\title{
Identification of Ornamental Plant Functioned as Medicinal Plant Based on Redundant Discrete Wavelet Transformation
}

\author{
Kohei Arai, Indra Nugraha Abdullah, Hiroshi Okumura \\ Graduate School of Science and Engineering \\ Saga University \\ Saga City, Japan
}

\begin{abstract}
Human has a duty to preserve the nature. One of the examples is preserving the ornamental plant. Huge economic value of plant trading, escalating esthetical value of one space and medicine efficacy that contained in a plant are some positive values from this plant. However, only few people know about its medicine efficacy. Considering the easiness to obtain and the medicine efficacy, this plant should be an initial treatment of a simple disease or option towards chemical based medicines. In order to let people get acquaint, we need a system that can proper identify this plant. Therefore, we propose to build a system based on Redundant Discrete Wavelet Transformation (RDWT) through its leaf. Since its character is translation invariant that able to produce some robust features to identify ornamental plant. This system was successfully resulting $95.83 \%$ of correct classification rate.
\end{abstract}

Keywords - Identification; ornamental plant; leaf; wavelet; DWT; Redundant DWT; SVM.

\section{INTRODUCTION}

All living things in this world need oxygen. Plant has an important role to produce oxygen and supply it to them. The cycle between human and the plant is the interesting one. Human respiration resulting carbon dioxide that needed by plant and as a result of photosynthesis plant assembled oxygen that vital for human. According to that cycle, human supposed to preserve the plant to maintain availability of oxygen.

Apart from as the producer of oxygen, some plants also have medicine efficacy. In Japan, medicinal plant has been developing until now, under Research Center for Medicinal Plant Resources. That research center consists of four divisions, start from Hokkaido Division, Tsukuba Division, Wakayama Branch and Tanegashima Division. Each Division has particular features and representative plants. Similar with Japan, Indonesia also has a history about medicinal plants. It starts from Jacobus Rontius in 1592-1631 announced 60 plants which had medicinal function. He wrote to his book titled $D e$ Indiae Untriusquere Naturali et Medica followed by in 1888 established Chemis Pharmalogisch Laboratorium as a part from Bogor Botanical Garden to investigate ingredients from medicinal plants. Currently, many researches about medicinal plants have been conducting under Indonesian Science Board.

Unfortunately, only few people especially young people that acquaint with ornamental plant functioned as a medicinal plant. Elderly people in Indonesia had known its medicine efficacy when the chemical based medicine did not yet attained the popularity. Also, It is easy to find because usually cultivates in front of the house. However, their common purpose of cultivating is to escalating esthetical value only, whereas many simple diseases can be cured using it.

We need an identification system to support people to know more about ornamental plant functioned as a medicinal plant. In this research, we propose to use leaf as identification object of that plant. The main reason is a leaf from ornamental leaf not depends on the season, dissimilar with flower that totally depends on the season.

Leaf identification can be done through identification of leaf shape and arrangement, leaf margin, or leaf venation. This identification is not a current instance in image processing area shown with numbers of researches conducted it. However, most of the works involved features from leaf venation as distinctive value of one leaf with others. One of the work that proposed by Park, Hwang, and Nam [1]. They were developing leaf image retrieval system using four typical venation types from plants collection in Korea. Their work can be separated to be two main steps. First, point selection as representation of leaf venation selected by Curvature Scale Scope Corner Detection. Second, selected points consist of branching point and end point was categorized by calculating density function of feature points.

Other work that related with this research is from Wang et.al [2]. They proposed a system by using automatic markercontrolled watershed segmentation method, as shape features representation they were utilizing $7 \mathrm{Hu}$ geometric moments and 16 Zernike moments. Their work was resulting $92.6 \%$ of average correct classification rate.

\section{PROPOSED METHOD}

\section{A. Dataset}

The dataset is leaf dataset and obtained by direct acquisition from ornamental plants collection in Indonesia. It consists of 8 classes, and each class has 15 images. Size of the image is $256 \times 256$ pixels. The classes are Bay (syzygium polyanthum), Cananga (canagium odoratum, lamk), Mangkokan (nothopanax scutellarium merr.), Jasmine (jasminum sambac [soland]), Cocor bebek (kalanchoe 
pinnuta), Vinca (catharanthus roseus), Kestuba (euphorbia pulcherrima, willd), Gardenia (gardenia augusta, merr).

Identification of leaf can be done through extraction of three features information. First, from the shape and arrangement, second is margin, and last is venation. Scope of this research covered various types of these features that represent the data set. Elliptic, reinform, obtuse, and deltoid types as shape and arrangements feature; dentate, entire, and undulate types as margin feature; pinnate and arcuate types as venation feature.

In order to eliminate unnecessary area outside the leaf, this research proposes segmentation process using Otsu's threshold method. Otsu's threshold calculates threshold to separate foreground and background with minimal intra-class variance. The following is sample images:
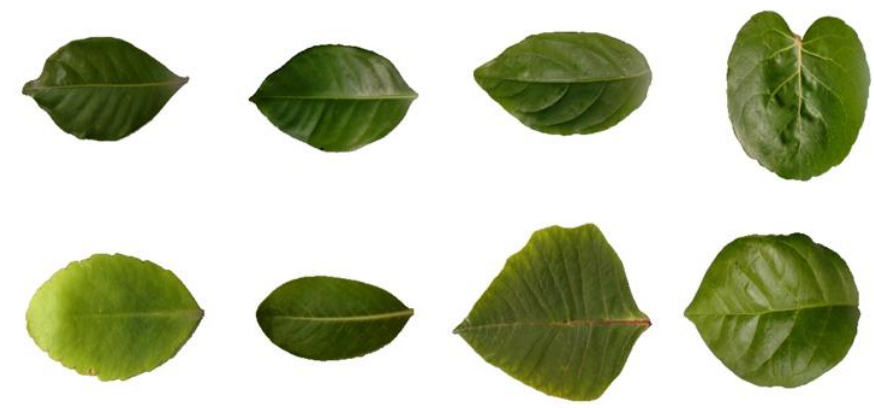

Fig. 1. Leaf images in the dataset.

TABLE I. MEDICINE EFFICACY CONTAINED IN ORNAMENTAL PLANT.

\begin{tabular}{|l|l|}
\hline Leaf's name & \multicolumn{1}{|c|}{ Medicine Efficacy } \\
\hline Bay & Diarrhea, scabies and itching, gastric ulcer. \\
\hline Cananga & Asthma, bronchitis. \\
\hline Mangkokan & Mastitis, skin injury, hair loss. \\
\hline Jasmine & Sore eyes, fever, head ache. \\
\hline Cocor Bebek & Hemorrhoids, sore joints. \\
\hline Kestuba & Bruise, irregular menstrual, dysentery. \\
\hline Gardenia & Diabetes, sprue, constipation. \\
\hline
\end{tabular}

From Table 1, numbers of ordinary diseases can be cured using the corresponding leaf. Those medicine efficacies can be gained in many serving ways. Most of them are the leaves boil together in the water and apply as a drink. Another way i.e. for skin injuries, only put the mangkokan leaf into the injury area [3].

\section{B. Redundant Discrete Wavelet Transformation (RDWT)}

Primary point of RDWT usage is because including in translation invariant family of wavelet transformation. The odd and even pixels are used for approximation and detail coefficients. Distinct with the other RDWT, in this research we decompose the input image with size exactly same, it means there is no frame expansion.

Based on above image, unlike the ordinary DWT the RDWT utilize all pixels in the image. The odd pixel is for scaling coefficient, and the even pixel is for wavelet coefficient. However, these methods still using down sample by 2 when convolves around the image. We use the spline wavelet filter which has one vanishing moment.

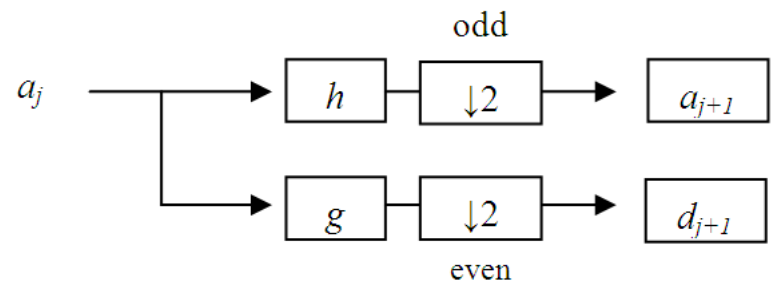

Fig. 2. Forward transformation of RDWT.

The scaling function on the RDWT is very similar with DWT is denoted by $\phi\left(a_{1}, a_{2}\right)=\phi\left(a_{1}\right) \phi\left(a_{2}\right)$. The part from one resolution to next is represented by:

$$
\begin{gathered}
c_{j+1}[k, l]=\sum_{m, n} h[m-2 k] h[n-2 l] c_{j}[m, n] \\
=\left[\overline{h h} * c_{j}\right]_{\downarrow 2,2}[k, l]
\end{gathered}
$$

The $\downarrow 2,2$ is defined as the down sample by factor of 2 along $\mathrm{x}$ and $\mathrm{y}$ axes. However, as aforementioned this method for scaling coefficient will keep the odd pixel and for wavelet coefficient will keep the even pixel. That is the reason the extension of DWT is called redundant.

The detail coefficient images from three sub wavelets:

Vertical wavelet: $\psi^{1}\left(a_{1}, a_{2}\right)=\phi\left(a_{1}\right) \psi\left(a_{2}\right)$

Horizontal wavelet: $\psi^{2}\left(a_{1}, a_{2}\right)=\psi\left(a_{1}\right) \phi\left(a_{2}\right)$

Diagonal Wavelet: $\psi^{3}\left(a_{1}, a_{2}\right)=\psi\left(a_{1}\right) \psi\left(a_{2}\right)$

In this research, we are also comparing the RDWT method to other methods the CDF 5/3 via lifting and Daubechies DB-4 methods. Lifting method in wavelet transformation supports some advantages. It allows us faster implementation of the wavelet transform, fully in-place calculation, the inverse wavelet transform only by do reverse action toward the decomposition wavelet transform result [4]. CDF 5/3 via lifting based on bi-orthogonal wavelet base function resulting perfect reconstruction image. It utilizes symmetry coefficient between scaling and wavelet function. The main reason of choosing Daubechies orthogonal of length four is needs lowcomputation time comparing to other wavelets in the Daubechies orthogonal family. We are expecting the competitive results from those methods.

\section{Support Vector Machine (SVM)}

SVM is a powerful tool for data classification. The indicators are the easiness to apply and impose Structural Risk Minimization (SRM). SRM armed the SVM to have strong ability in generalization of data. Its function is to minimize an upper bound on the expected risk. In principle, SVM learns to obtain optimal boundary with maximum margin that able to separate set of objects with different class of membership.

In order to achieve the maximum margin classifier, we have two options. Hard margin and soft margin are the options that totally depend on linearity of the data. Hard margin SVM 
is applicable to a linearly separable dataset. However, often the data is not linearly separable. Soft margin SVM emerged as its solution [5]. The optimization problem for the soft margin SVM presented as below:

$$
\begin{gathered}
\min _{w, b} \frac{1}{2}\|w\|^{2}+C \sum_{i=1}^{n} \xi_{i} \\
\text { subject to: } \quad y_{i}\left(w^{T} x_{i}+b\right) \geq 1-\xi_{i}, \quad \xi_{i} \geq 0 .
\end{gathered}
$$

where $w, C, \xi, b$ are the weight vectors, the penalty of misclassification or margin errors, the margin error, the bias, respectively.

In (2) can lead us to efficient kernel methods approach. A kernel method is an algorithm that depends on the data only through kernel function, which computes a dot product in some possibly high dimensional data.

Using the function $\phi$ training vector the input space $x$ is mapped into higher dimensional space. $K\left(x_{i}, x_{j}\right)=$ $\phi\left(x_{i}\right)^{T} \phi\left(x_{j}\right)$ is called kernel function. The degree of the polynomial kernel can control the flexibility of resulting classifier [6]. It will be appropriate with this research when we classify 8 types of leaf. Polynomial kernel is shown in equation (3).

$$
K\left(x_{i}, x_{j}\right)=\left(\gamma x_{i}^{T} x_{j}+r\right)^{d}, \gamma>0
$$

where $\gamma, r, d$ are kernel parameters, and $i, j$ denote $i^{t h}, j^{\text {th }}$ vector in dataset.

In this research, we propose to use Sequential Minimal Optimization (SMO). SMO act as efficient solver of the optimization problem in training of support vector machines. SMO also solves the problems analytically by way of breaks the problems into a series of smallest possible problems.

Despite of this algorithm guaranteed to converge, it used heuristics to choose the pair of multipliers that able to accelerate the rate of converge.

\section{EXPERIMENTS}

\section{A. Forward Transformation RDWT}

The small difference when keep the scaling and wavelet coefficient gave us expected result. The translation invariance feature guarantees the leaf venation is proper extracted from the image.

As decomposition result level 2 of RDWT, CDF 5/3 via lifting method as well as DB-4 are shown with the following images:

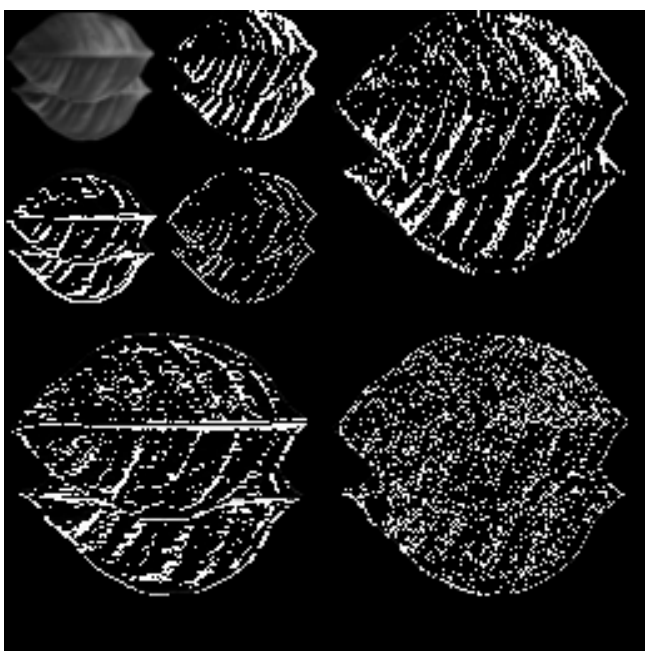

Fig. 3. Forward transformation result for RDWT.

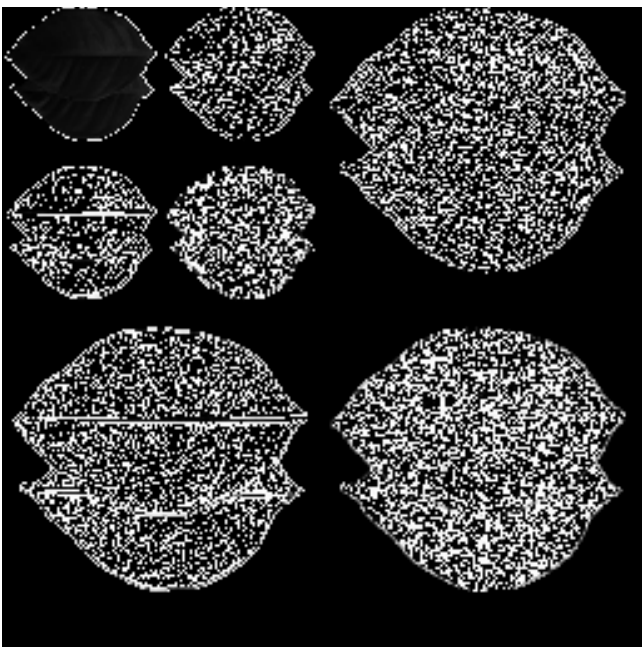

Fig. 4. Forward transformation result for $\mathrm{CDF} 5 / 3$.

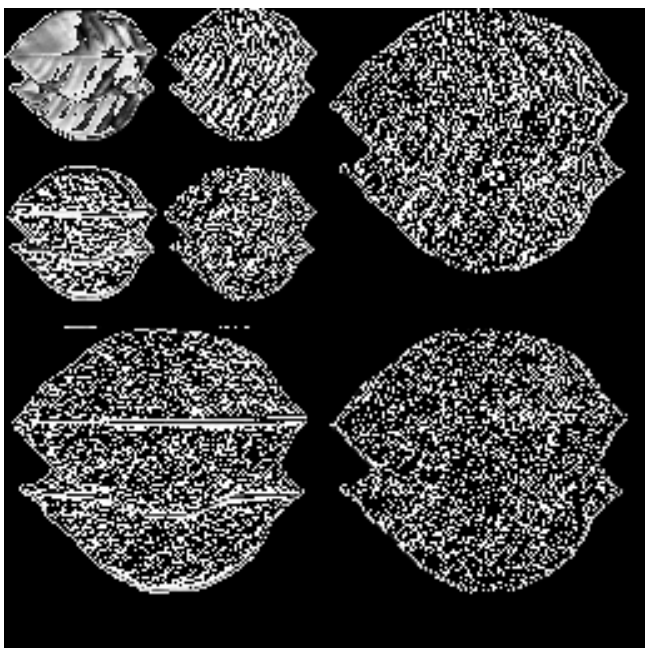

Fig. 5. Forward transformation result for DB-4. 
Those images indicate us that decomposition from RDWT is preferable compare to the CDF 5/3 method and DB-4. From the result, it is concluded that the RDWT successfully obtained much clear leaf venation as one crucial point to identify leaf than the CDF.

\section{B. Features Selection}

Features selection is based on statistical theory consist of total, mean, variance, and standard deviation. These features capture from decomposition result of each detail, approximation, horizontal, vertical, and diagonal details, visualize as shown in Figure 6.

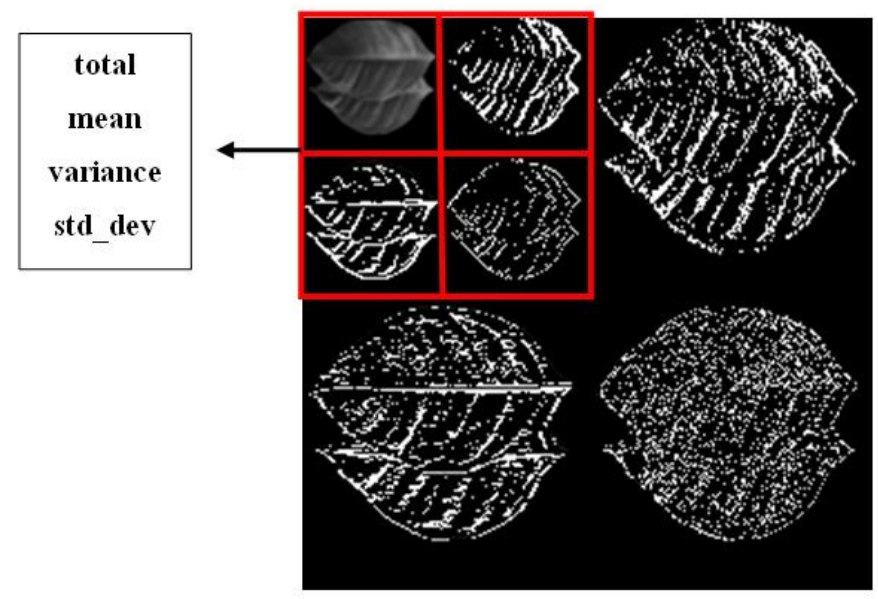

Fig. 6. Features selection.

The mean, variance, and standard deviation are valuable because of their relationships to the normal curve. Variance distribution represents the high frequency data that related with leaf venation. Likewise, total number of wavelet coefficient involved as an additional feature to represent high frequency data. Approximation included because some of distinct factor that contained in the leaf, e.g. leaf shape and arrangement from leaf can be obtained from the approximation coefficient.

\section{Classification Results}

Data proportion between training and testing data are $80 \%$ and $20 \%$, respectively. It means 12 images from each class act as training data and 3 images act as testing data. Table 2, 3, and 4 show the classification performance of three aforementioned methods.

TABLE II. PERFORMANCE RESULT OF LEAF IDENTIFICATION THROUGH WAVELET TRANSFORMATION FOR ALL DETAILS (IN PERCENT)

\begin{tabular}{|l|c|c|c|}
\hline \multirow{2}{*}{ Method } & \multicolumn{3}{|c|}{ L, H, V, D } \\
\cline { 2 - 4 } & RDWT & CDF 5/3 & DB-4 \\
\hline Training set & 97.92 & 96.88 & 97.92 \\
\hline Supplied test set & 95.83 & 87.5 & 91.67 \\
\hline
\end{tabular}

TABLE III. PERFORMANCE RESULT OF LEAF IDENTIFICATION THROUGH WAVELET TRANSFORMATION FROM THREE HIGH FREQUENCY DETAILS (IN PERCENT)

\begin{tabular}{|l|c|c|c|}
\hline \multirow{2}{*}{ Method } & \multicolumn{3}{|c|}{ H, V, D } \\
\cline { 2 - 4 } & $\boldsymbol{R D W T}$ & $\boldsymbol{C D F}$ 5/3 & $\boldsymbol{D B}-\mathbf{4}$ \\
\hline Training set & 92.71 & 83.33 & 78.13 \\
\hline Supplied test set & 79.17 & 66.67 & 66.67 \\
\hline
\end{tabular}

TABLE IV. PERFORMANCE RESULT OF LEAF IDENTIFICATION THROUGH WAVELET TRANSFORMATION FROM HORIZONTAL AND VERTICAL DETAILS (IN PERCENT)

\begin{tabular}{|l|c|c|c|}
\hline \multirow{2}{*}{ Method } & \multicolumn{3}{|c|}{ H, V } \\
\cline { 2 - 4 } & $\boldsymbol{R D W T}$ & $\boldsymbol{C D F}$ 5/3 & $\boldsymbol{D B} \mathbf{- 4}$ \\
\hline Training set & 82.29 & 73.96 & 72.92 \\
\hline Supplied test set & 70.83 & 58.33 & 58.33 \\
\hline
\end{tabular}

L, H, V, D denotes approximation detail, horizontal detail, vertical detail and diagonal detail, respectively. We decided to present only $\{\mathrm{L}, \mathrm{H}, \mathrm{V}, \mathrm{D}\}, \quad\{\mathrm{H}, \mathrm{V}, \mathrm{D}\}, \quad$ and $\{\mathrm{H}, \mathrm{V}\} \quad$ as representation of previously mentioned leaf features. High frequency details as well as low frequency detail are needed for the identification, when D detail consists high frequency data only. Performance over the training set shows us how well the SOM learned the data.

Linear classifiers often have simple training algorithms that fit with the number of samples. However, better accuracy is provided by non-linear classifier in many applications. Identification through classification by SMO in table 1 yielded the positive results indicated by all the performances are around $90 \%$. The distribution from 8 classes data attained well separated decision boundary by the non-linear SVM kernel method, in this case is through polynomial kernel function.

The CDF 5/3 and DB-4 in table 2 showed deficient performances with $58.33 \%$ of correct classification results. Similar performances showed in table 3 these methods had same values, which was $66.67 \%$. We concluded that the CDF $5 / 3$ and DB-4 had identical characteristics while extracting the feature which are clearly visible as shown in Figure 5 and 6.

From Table 2 and 3, the approximation detail provided significant improvement. It is obvious seen in the supplied test set from the $\{\mathrm{L}, \mathrm{H}, \mathrm{V}, \mathrm{D}\}$ and $\{\mathrm{H}, \mathrm{V}, \mathrm{D}\}$, we gained $9.52 \%$, $13.52 \%$, and $15.79 \%$ of improvement from RDWT, CDF5/3, and DB-4, respectively. It implied that ornamental leaf feature also occurred in here. In the sequel, at the supplied test result also obtained the result of RDWT is better than CDF 5,3 and DB-4. Utilization of all pixels in RDWT's behavior can extract clearly of leaf venation as well as texture feature, when the other methods only extract texture feature from leaf.

Misclassification for each method often happened in class $A$ and B. The reason was class A and B had same characteristics from the shape and arrangement as well as leaf venation. 
Even though, we were involving human perception, often made a mistake while trying to classify those classes. It will be the future study to select another appropriate wavelet transformation's feature representation from the leaf.

\section{CONCLUSION}

This research has successfully conducted RDWT to extract relevant leaf information. This modified version of DWT in utilization of odd and even pixels was resulting preferable result in comparison with CDF 5/3 via lifting and Daubechies DB-4 methods. In general, feature selection in this proposed method also in the proper way has represented the leaf information through its leaf venation and shape. Though we were working on different dataset, obtained correct classification result of $95.83 \%$ is greater than Wang et.al work. Even we gained the proper classification performance. On the other hand, we are still facing another serious problem. Another problem is how we can educate and motivate people after they get acquainted to use this ornamental leaf as an alternative of treatment from one disease. Since the selfdosage thing will not be a cure, but aggravate the disease even worse.

\section{FUTURE WORK}

The future work that can be interesting from this current progress is conducting the other wavelet transformation method with better support of contour extraction. One of the promising candidates is dyadic wavelet transformation via lifting method. Frame expansion and shift invariant features are the guarantee points to obtain better contour extraction. The other future study is an extension from this basic feature extraction in relation with complexity of the dataset.

\section{ACKNOWLEDGMENT}

This research delivered under cooperation between Department of Information Science, Saga University, Japan and Department of Computer Science, Bogor Agricultural Institute, Indonesia.

\section{REFERENCES}

[1] Park Jinkyu, Hwang Eenjun, Yunyoung Nam, "Utilizing venation features for efficient leaf image retrieval", The Journal of System and Software, Vol.81, pp. 71-82, 2008.
[2] Wang Xiao Feng, et.al, "Classification of plant images with complicated background", Applied Mathematics and Computation, Vol.205, pp. 916926, 2008.

[3] Hartati Sri, "Tanaman hias berkhasiat obat", IPB Press, 2011. (In Indonesian)

[4] Sweldens Wim, "Wavelet and the lifting scheme: a 5 minute tour".

[5] Ben-Hur Asa, Weston Jason, "A user's guide to support vector machine", in Data Mining Techniques for the Life Science, pp 223-239, Humana Press, 2010.

[6] Hsu Chih-Wei, Chang Chih-Chuang, Lin, Chih-Jen, "A practical guide to support vector classification".

[7] Starck Jean-Luc, Murtagh Fionn, Fadili Jalal M, "Sparse image and signal processing", Cambridge University Press, 2010.

[8] Mallat Stephane, "A wavelet tour of signal processing", Academic Press, 2009.

\section{AUTHORS PROFILE}

KOHEI ARAI received BS, MS and PhD degrees in 1972, 1974 and 1982, respectively. He was with The Institute for Industrial Science and Technology of the University of Tokyo from April 1974 to December 1978 and also was with National Space Development Agency of Japan from January, 1979 to March, 1990. During from 1985 to 1987, he was with Canada Centre for Remote Sensing as a Post Doctoral Fellow of National Science and Engineering Research Council of Canada. He moved to Saga University as a Professor in Department of Information Science on April 1990. He was a counselor for the Aeronautics and Space related to the Technology Committee of the Ministry of Science and Technology from 1998 to 2000. He was a counselor of Saga University for 2002 and 2003. He also was an executive counselor for the Remote Sensing Society of Japan for 2003 to 2005. He is an Adjunct Professor of University of Arizona, USA since 1998. He also is Vice Chairman of the Commission A of ICSU/COSPAR since 2008 .

INDRA NUGRAHA ABDULLAH was born in Bogor, Indonesia on June $18^{\text {th }}$ 1987. Finished his bachelor degree in Bogor Agricultural University and graduated from Saga University for master degree in the field of Information Science on March 2011. He is currently pursuing to get Ph.D. Degree from the same university with specialization in image processing area. Leaf identification becomes his interest in his latest degree.

HIROSHI OKUMURA was born at Kyoto, Japan in 1964. He received B.E.S.E. and M.E.S.E. degree from Hosei University in 1988 and 1990, respectively, and $\mathrm{Ph} . \mathrm{D}$ degree on environmental engineering from Chiba University in 1993. He became a research associate at Remote Sensing and Image Research Center, Chiba University first in 1993. Next, he became a research associate and a lecturer at the Department of Electrical Engineering, Nagaoka University of Technology in 1995 and 2000, respectively. He is now an associate professor at the Department of Information Science, Saga University. His research interests are in image and speech processing and remote sensing. 